\title{
A New Experimental Approach for In Situ Damage Assessment in Fibrous Ceramic Matrix Composites at High Temperature
}

\author{
Jared Tracy, ${ }^{\star \dagger}$ Anthony Waas, ${ }^{\S}$ and Samantha Daly" \\ ${ }^{\star}$ Materials Science and Engineering, University of Michigan, 3636 GGB Building, 2350 Hayward, Ann Arbor, Michigan 48109

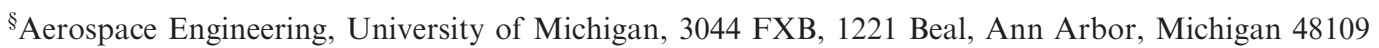 \\ ${ }^{\top}$ Mechanical Engineering, University of Michigan, 3674 GGB Building, 2350 Hayward, Ann Arbor, Michigan 48109
}

\begin{abstract}
High-temperature applications of ceramic matrix composites necessitate a rigorous understanding of the fracture and damage mechanisms that occur under thermomechanical loading, requiring the development of advanced small-scale characterization approaches. In this work, fiber-reinforced $\mathrm{SiC} / \mathrm{SiC}$ tensile specimens were loaded in a scanning electron microscope at $800^{\circ} \mathrm{C}$, and full-field deformation maps at the constituent length scale were generated using digital image correlation (DIC). A colloidal system containing mechanically milled titanium nanopowder, bicine, and water was developed for use as a DIC tracking pattern that is stable at $795^{\circ} \mathrm{C}$. The resultant full-field strain maps provide a constituent level characterization of damage evolution from crack initiation through final fracture. An analysis of strain along fiber lengths indicated that fiber mean strain and standard deviation reached a minimum at fiber fracture. In addition, multiple matrix cracks in the process zone ahead of a notch/crack tip were apparent and could falsely appear as a continuous region of high strain in DIC fields. Relatively large displacement (strain) error was attributed to noise and bias at these small length scales and small strain values, and approaches for mitigating this error are discussed.
\end{abstract}

\section{Introduction}

$\mathrm{T}$ HE next generation of high-temperature ceramic composites for aerospace applications will experience more rigorous thermomechanical loading than their superalloy counterparts currently in service. Ceramic matrix composites (CMCs) are lighter than superalloys while maintaining their structural integrity at higher temperatures, desirable qualities for improving aircraft engine efficiency. The $\mathrm{SiC} / \mathrm{SiC}$ materials system is relatively new; although there exist analytical and numerical models to predict its mechanical behavior at elevated temperature, experimental information beyond grossly averaged macroscopic data is needed for rigorous model validation and an improved understanding of damage and toughening mechanisms. This study describes a novel approach to probe damage progression in CMCs at high temperatures and to quantify toughening mechanisms at the constituent scale.

Several researchers have investigated the micromechanics of deformation and fracture in CMCs. Aveston, Cooper and Kelly ${ }^{1}$ developed an analytical model (ACK) characterizing matrix cracking stress and interfacial shear stress in a unidirectional CMC. Marshall, Cox, and Evans ${ }^{2}$ attributed tough-

C.-H. Hsueh-contributing editor

Manuscript No. 35116. Received June 16, 2014; approved February 2, 2015.

${ }^{\dagger}$ Author to whom correspondence should be addressed. e-mail: jmtracy@umich.edu ening to regularly spaced matrix cracks bridged by uniaxially aligned fibers, and proposed analytical models $(\mathrm{MCH})$ suggesting that the steady-state cracking stress depends on composite microstructure. The two models were compared by Hsueh, ${ }^{3}$ who noted that their assumptions about crack displacement had different physical meanings. Budiansky, Hutchinson, and Evans ${ }^{4}$ likewise proposed an analytical model for weakly bonded fiber-matrix interfaces that predicted a critical stress for cracking based on the debond lengths of fibers subjected to matrix cracks; here, toughening was attributed to frictional resistance at the debonded interface. Investigating fracture in $\mathrm{SiC}$ whisker-reinforced ceramics, Becher ${ }^{5}$ characterized the contributions of debonding and pullout to toughening. $\mathrm{Zok}^{6}$ attributed toughening in CMCs to fiber bridging tractions and fiber pullout, with its degree dependent on a characteristic bridging zone length behind the propagating crack tip. From a micromechanics perspective, fracture resistance depends on frictional sliding resistance at the fiber-matrix interface as well as the length of the intact fiber. ${ }^{6}$ Dassios and Galiotis ${ }^{7}$ devised a clever technique to measure the toughening contribution of fibers using Raman spectroscopy. Strains in the constituent fibers of a $\mathrm{SiC} /$ glass-ceramic CMC where measured in situ under tension upon initiation of the first matrix crack, then tensile and interfacial stresses were backed out. However, their CMC had a much more brittle matrix crack than the $\mathrm{SiC} / \mathrm{SiC}$ CMCs in this work, and large scale bridging occurred immediately after crack initiation. Although reputable analytical models exist to explain toughening in CMCs, little is known about crack nucleation and propagation at the constituent level at elevated temperature.

Full-field deformation measurements at the constituent length scale can improve our quantitative understanding of damage onset in CMCs. These can be captured through the judicious use of digital image correlation (DIC), which is a noncontact method of measuring full-field deformations on the surface of a material by calculating the relative displacements between features of a tracking pattern. Using a subsetmatching algorithm, deformation fields are calculated from sequential images of the pattern taken during thermomechanical loading. Early work in DIC focused on optimizing its accuracy by removing the spatial distortions inherent in optical imaging equipment. ${ }^{8,9}$ Recently, the development of spatial and temporal image distortion corrections, coupled with advances in small-scale patterning, have expanded DIC to high magnification SEM imaging. ${ }^{9-19}$ The combination of SEM and DIC (termed here as SEM-DIC), with customdeveloped patterning and distortion correction procedures, was used to investigate the small-scale mechanical behavior of a CMC.

SEM-DIC accuracy strongly depends on tracking pattern quality. Several studies have addressed generating high-contrast, isotropic patterns on the surface of various materials at 
microscopic and macroscopic length scales..$^{8,9,20,21}$ A successful means for small-scale patterning utilizes the self-assembly of gold and silver nanoparticles, ${ }^{15,19,22-24}$ but these metals are not suitable for elevated temperatures. E-beam patterning can produce adequate patterns using a variety of temperature resistant metals and ceramics, ${ }^{16}$ but it requires a perfectly flat surface and is too time-consuming and expensive for fracture investigations where uncertainty in the crack path requires that the pattern cover a large area. At high temperatures, a good patterning alternative is nanopowder (NP) deposition utilizing high-temperature mediums like Ti and WC. Because nanopowders naturally aggregate both in powder form and in suspension, the particles must be stabilized in a colloidal dispersion. Identifying dispersants, surfactants or stabilizers that minimize aggregation is critical. WC has been shown to form a stable colloid in water alone, ${ }^{25}$ but incorporation of dispersants or buffers can further reduce aggregation. ${ }^{26-29}$ Mechanical milling (roll-milling and sonication) enhances the effectiveness of dispersants by breaking down large clumps and agglomerates.

The combination of scanning electron microscopy and digital image correlation (SEM-DIC) was used to quantitatively characterize deformation at the constituent length scale in CMC test specimens. SEM-DIC has limitations: currently it is constrained to 2-D deformation mapping and it is slower than optical imaging. Working within these limitations, it is a unique and powerful approach for investigating heterogeneous deformations in multiphase material at the constituent length scale. This approach, detailed by Sutton et al. in $2007,{ }^{10,11}$ has recently been used to probe microscale mechanical behavior in fiber-reinforced composites. ${ }^{14,30}$ In 2012, Canal et al. ${ }^{14}$ found that DIC subset size affects the ability to capture sharp displacement gradients that exist at the fiber-matrix interface. At magnifications high enough that the subset size was less than $20 \%$ of the fiber diameter, the average strains computed in each constituent were qualitatively equivalent to numerical model predictions; that is, the average strains were smaller in transversely loaded fibers than in the matrix. However, the error between the finite element and DIC predictions was large. ${ }^{14}$ For both large and small magnifications, average strains in the composite matched (to within $300 \mu \varepsilon$ ) the expected value based on the applied stress and known elastic constants.

To date, the DIC characterization of CMCs has focused on either ambient temperature studies or elevated temperature macroscale studies. ${ }^{20,21}$ Utilizing SEM-DIC on ceramic composites presents new and complex challenges. These stem from the fact that as the field of view (FOV) decreases, it encompasses a lower density of matrix cracks. Portions of the FOV that fall outside of the process zone of a crack contain only elastic deformations, where noise and bias induced error can mask strain measurements. However, if caution is used to minimize potential error sources, SEM-DIC is a remarkably useful tool for assessing deformation and damage in CMCs.

SEM-DIC is also advantageous in that thermal turbulence (heat haze) is a nonissue under a vacuum environment. Novak $^{21}$ macroscopically characterized C-SiC CMCs at $1450^{\circ} \mathrm{C}$ in air, noting that a major challenge was minimizing heat haze and its detrimental effect on image distortion. Although spatial and temporal image distortions resulting from electron beam rastering should be considered when using SEMDIC, these can be successfully accounted for. ${ }^{11-13,15}$ The SEM must also be appropriately shielded to contain thermal radiation; the importance of this is illustrated in supplementary material and discussed herein.

This study presents an approach for measuring full-field deformations at the microscale in $\mathrm{SiC} / \mathrm{SiC} \mathrm{CMCs}$ under thermomechanical loading, using a combination of digital image correlation and scanning electron microscopy (SEM-DIC). New nanopowder DIC tracking patterns for elevated temperatures and the effect of DIC parameters on damage detection are discussed. Crack evolution was monitored in edge-notched $\mathrm{CMC}$ tensile coupons and revealed toughening mechanisms characteristic of fibrous ceramic composites. A framework for extending in situ, small-scale thermomechanical loading experiments to temperatures well above $1000^{\circ} \mathrm{C}$ is provided.

\section{Experimental Procedure}

\section{(1) Materials Preparation and Patterning}

Two 8-ply $[0 / 90 / 0 / 90]_{\text {s }}$ melt-infiltrated $\mathrm{SiC} / \mathrm{SiC}$ composite laminates were machined into single edge-notch tensile coupons with the dimensions shown in Fig. 1(a). Notches were cut with a Mager diamond wafering blade as shown in Fig. 1(b). The processing method is intellectual property of the manufacturer and will not be discussed in this study. However, general discussions of processing of $\mathrm{SiC} / \mathrm{SiC} \mathrm{CMCs}$ can be found in relevant literature. ${ }^{31-33}$ Specimens were polished using 9, 3, and $1 \mu \mathrm{m}$ diamond lapping films (Buehler) to prevent nanopowders from aggregating in rough surface features and to create a uniformly flat surface. To ensure uniform removal of material during lapping and polishing, coupons were encased in an acrylic sacrificial mold. Upon completion of polishing, coupons were separated from the mold and agitated in a sonicator, in both water and ethanol, in preparation for patterning.

All tests were conducted at $795^{\circ} \mathrm{C}$ due to a heater limitation of $800^{\circ} \mathrm{C}$. Ti was used for patterning as it forms a welldispersed pattern at this temperature; note that above $\sim 1000^{\circ} \mathrm{C}$, Ti reacts with the underlying $\mathrm{SiC}$ to form a silicide, degrading pattern quality and possibly degrading the mechanical properties of the composite. For testing above $\sim 1000^{\circ} \mathrm{C}$, WC is a better choice for patterning $\mathrm{SiC}$. The $\mathrm{Ti}$ NP suspension contained water and bicine; the bicine served as a $\mathrm{pH}$ buffer to stabilize the colloid. Figure 2 shows a $\mathrm{SiC}$ substrate coated with the Ti colloidal suspension. Tracking patterns were applied by drop casting approximately $0.5 \mu \mathrm{L}$ droplets of $\mathrm{Ti}$ nanopowder colloid onto the surface of the tensile specimen. The colloidal suspension contained $22 \mathrm{mg}$ of bicine and $3 \mathrm{mg}$ of $80 \mathrm{~nm}$ titanium powder in $1 \mathrm{~mL}$ of distilled water. The suspension was roll-milled for $24 \mathrm{~h}$ prior to drop casting to break up agglomerates. The combination of roll-milling and $\mathrm{pH}$ buffering broke up the heavily aggregated nanopowder. Deposition droplets were applied at the notch and throughout the width of the sample. Upon

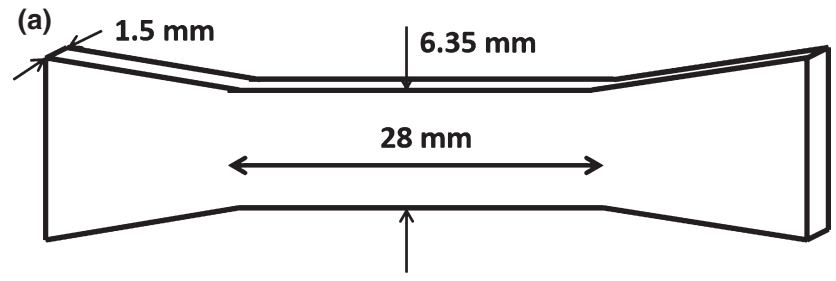

(b)

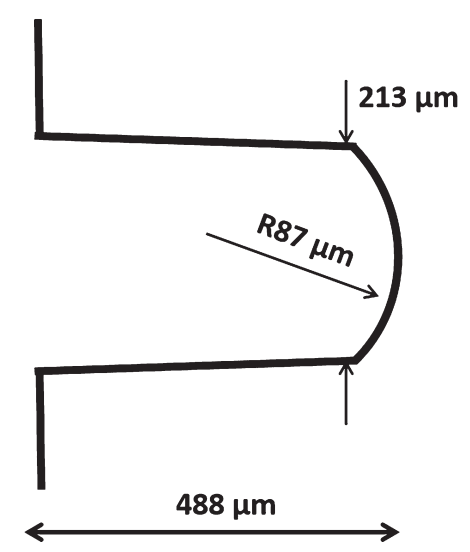

Fig. 1. Dimensions of (a) tensile coupon and (b) edge notch. 


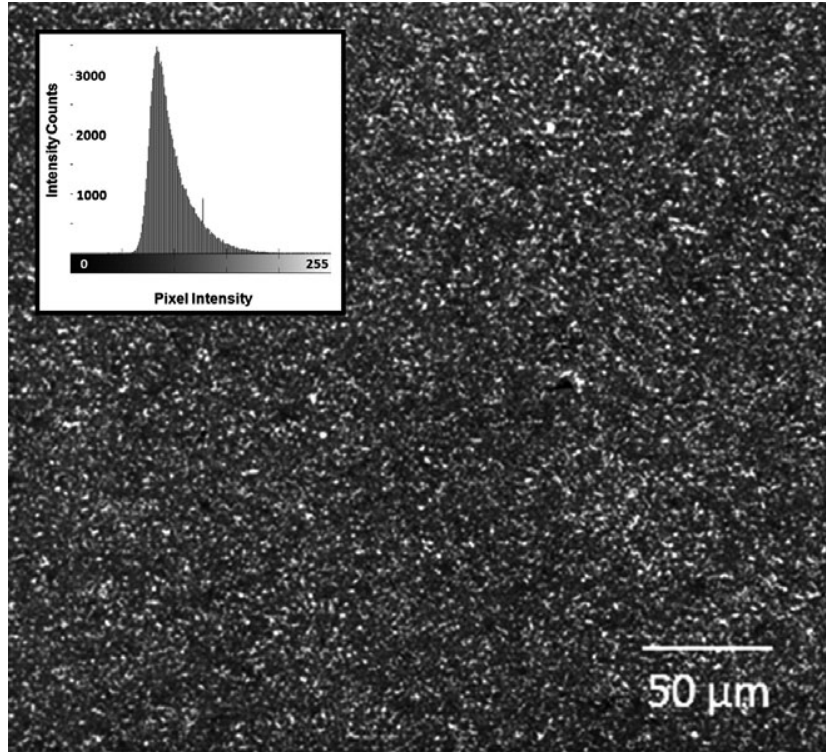

Fig. 2. High-temperature DIC tracking pattern for tests at $795^{\circ} \mathrm{C}$, consisting of slightly aggregated $80 \mathrm{~nm} \mathrm{Ti}$ nanopowder on a $\mathrm{SiC}$ substrate and applied by drop casting. A grayscale pattern intensity histogram is shown in the upper left indicating good pattern quality.

evaporation, the sample was heated to $300^{\circ} \mathrm{C}$ on a hot plate to burn off the bicine. The resulting tracking patterns for the two test coupons are provided as supplementary material in Fig. S1.

Pattern quality is a critical factor in the accuracy of DICcalculated displacement fields. Poor patterns can result in incomplete correlations and introduce irremovable variability in the deformation data. In addition to error that can arise from poor-quality tracking patterns, there is inherent Gaussian noise in SEM images arising from multiple sources: mechanical vibrations, out-gassing and volatilization of foreign matter, and the statistical nature of electron interactions with the surface may all manifest as noise in DIC data. To confirm that the patterns were suitable for SEM-DIC, static image pair and rigid body translation images were captured in the SEM and postprocessed to significantly reduce spatial and temporal image distortions with a combination of laboratory-developed code and commercial code. ${ }^{34}$ For details on SEM-DIC distortion corrections, please refer to relevant publications. ${ }^{11-13}$ Although this work was conducted at high temperature, pattern quality was examined at ambient temperature as testing determined that pattern quality did not degrade at high temperature.

\section{(2) High-Temperature SEM-DIC}

High-temperature testing was conducted in a FEI (Hillsboro, OR) Quanta 3D scanning electron microscope using an in situ tensile stage and heater (Kammrath \& Weiss, Dortmund, Germany); the experimental setup is provided as supplementary material in Fig. S2(a). To prevent thermal emissions from flooding the SEM scintillator, a double layer of tantalum heat shields with small apertures for beam access was fitted above the sample as depicted in Fig. S2(b). Also included as supplementary material, Fig. S3 shows the dra- matic reduction in thermal radiation achieved at $795^{\circ} \mathrm{C}$ with heat shielding. To monitor the effect of stage heating and prevent SEM damage, four type-K thermocouples were placed on the load cell, SEM stage, near the pole piece and near the EDAX detector. The heater was composed of a heating element encased in alumina upon which sat a steel thermocouple platen. To improve the efficiency of heat transfer from the steel to the $\mathrm{SiC}$ tensile coupon under vacuum, a compliant graphite foil was sandwiched between the two surfaces.

Testing on the first tensile coupon, identified as "HT-1", was conducted using a $500 \mu \mathrm{m}$ FOV. For a second test on a different tensile coupon, identified as "HT-2", an $800 \mu \mathrm{m}$ FOV was used. Each micrograph was $2048 \times 1768$ pixels. Images were captured in secondary electron mode using a $30 \mathrm{kV}$ accelerating voltage and $2.6 \mathrm{nAmp}$ beam current at a frame rate of $36.3 \mathrm{~s} /$ frame, integrated four times. These parameters provided images with the highest contrast and lowest noise at the fastest imaging speed. A summary of test parameters is provided in Table I.

The samples were first heated to $795^{\circ} \mathrm{C}$ and then mechanically loaded while held at $795^{\circ} \mathrm{C} \pm 0.2^{\circ} \mathrm{C}$. Prior to heating, the samples were preloaded to $100 \mathrm{~N}$ and then relaxed to $0 \mathrm{~N}$ to remove slack and prevent slippage during the initial loading phase of testing. The unloaded samples were heated to just under $800^{\circ} \mathrm{C}$ in temperature increments of $100^{\circ} \mathrm{C}$. During each temperature increment, the load was continuously adjusted to maintain $0 \mathrm{~N}$ as the sample and grips expanded. At $500^{\circ} \mathrm{C}$ and every $100^{\circ} \mathrm{C}$ increment thereafter, contrast, brightness, and focus were adjusted to maintain a high quality image. After the sample stabilized at $795^{\circ} \mathrm{C}$, the LVDT was zeroed so that subsequent loading would start at zero displacement.

Specimens were loaded to $50 \mathrm{~N}$ and an additional $50 \mathrm{~N}$ at each increment under displacement control with a speed of $2 \mu \mathrm{m} / \mathrm{s}$. At each loading increment, the reading on the load cell was allowed one to two minutes (depending on the applied load and state of cracking) to stabilize before image capture. As crosshead displacement was held constant, the applied load dropped slightly. Although crack propagation likely contributes to this load drop, it could not be resolved visually. During the early stages of loading, the notch was imaged at higher magnifications at each load increment to identify crack initiation. Upon crack initiation, load increments were increased slightly to allow a small amount of crack propagation through the FOV.

Once the crack cleared the FOV, the stage was shifted to image the next speckle pattern ahead of the advancing crack. The sample was unloaded and a reference image was captured at a load of $0 \mathrm{~N}$. The sample was then reloaded and the process of capturing images continued. Unloading the sample to capture a reference image at a new position inadvertently introduced tensile load cycling, although not necessarily fatigue (which would require cycling at a fixed ratio). A definitive assessment of how load cycling affected mechanical performance could not be drawn from the limited data collected but will be addressed in future work. The goal of this work was to examine toughening mechanisms, in situ, during thermomechanical loading.

Although the tests were conducted at $795^{\circ} \mathrm{C}$, this was due to a physical limitation of the stage heater; the general approach can be extended to thermomechanical loading experiments well above $1000^{\circ} \mathrm{C}$. At higher temperatures,

Tables I. Summary of Parameters High Temperature Tensile Tests

\begin{tabular}{ccccccc}
\hline & $\begin{array}{c}\text { FOV } \\
(\mu \mathrm{m})\end{array}$ & $\begin{array}{c}\text { Image size } \\
(\text { pixels })\end{array}$ & $\begin{array}{c}\text { Image resolution } \\
(\mathrm{nm} / \text { pix })\end{array}$ & $\begin{array}{c}\text { DIC subset } \\
(\text { pixels })\end{array}$ & Displacement rate $(\mu \mathrm{m} / \mathrm{s})$ & Temperature $\left({ }^{\circ} \mathrm{C}\right)$ \\
\hline HT-1 & 500 & $2048 \times 1768$ & 244 & 51 & 2 & 795 \\
HT-2 & 800 & $2048 \times 1768$ & 391 & 51 & 2 & $71000 \mu \mathrm{mm}$ crack extension \\
\hline
\end{tabular}


materials that have high-melting temperatures and do not react with the sample (such as WC for SiC) should be used for patterning. The heating system should incorporate heat shields to reduce thermal noise and a cooling system to prevent overheating of the miniature load frame and SEM components.

\section{Results and Discussion}

\section{(1) High-Temperature Full-Field Deformations}

Figures 3 and 4 show in-SEM, full-field quantitative maps of damage progression as a crack initiates at a notch and propagates through a $\mathrm{CMC}$ at $795^{\circ} \mathrm{C}$. Of the two tests, one (HT2) was carried out to final fracture. Figure 3 shows the sequence of crack propagation and the corresponding longitudinal strain fields of test HT-2. To protect the proprietary nature of the test material, stress and strain values have been nondimensionalized in all figures. The small horizontal undulating variations in longitudinal strain magnitude are attributed to Gaussian salt-and-pepper noise and aliasing. DIC parameters (51 pixel subset and 71 pixel strain filter) were chosen to achieve the best displacement resolution possible without flooding the strain field with noise. The subset size was near that of the fiber diameter.

The DIC parameters used in this study do not collectively represent a rule that can be applied to all materials, but depend on speckle size, spatial resolution, image resolution, and the underlying microstructure. We chose a strain filter window approximately 1.5 times larger than the subset size because this relation has been shown to reduce strain error. ${ }^{20}$ However, the size of the subset should be the smallest that the speckle pattern permits without introducing aliasing. Subset size can be reduced by either increasing the pixel resolution of the image or using smaller nanoparticles at a larger magnification. For multiphase materials, it is desirable to use a subset smaller than the smallest constituent to reduce the effect of data smearing at the constituent interfaces.

Figure 4 compares longitudinal strain fields measured at fracture initiation from the notches for tests HT-1 and HT-2, both conducted in uniaxial tension at $795^{\circ} \mathrm{C}$ in-SEM: Fig. 4(a) shows a $500 \mu \mathrm{m}$ FOV (test HT-1) and Fig. 4(b) shows a $800 \mu \mathrm{m}$ FOV (test HT-2). Upon crack initiation in HT-2 [Fig. 4(b)], a small region of elevated longitudinal strain (as indicated by forked blue lobes) in the process zone is observed immediately ahead of the crack tip. In test HT-1 [Fig. 4(a)], a much shorter crack appears upon initiation, and emanating from the crack tip are two distinct lobes of strain in a larger process zone than of HT-2.

DIC parameter selection greatly influenced damage detection. For example, modifying DIC parameters for HT-1 (51 pixel subset and 375 pixel strain filter) as in Fig. 4(a) effectively smoothed over smaller, discrete regions of high strain that revealed the presence of additional matrix cracks.
To illustrate this difference, Fig. 5 shows the evolution of longitudinal strain with load for test HT-1 using two DIC parameter sets: Fig. 5(a) uses a subset of 51 pixels and strain filter of 375 pixels, whereas Fig. 5(b) uses a subset of 51 pixels and a strain filter of 75 pixels. Although the strain fields of [Fig. 5(b)] are substantially noisier than [Fig. 5(a)], they reveal four distinct matrix cracks (indicated by red contours) emanating from the notch tip. The larger strain filter employed in [Fig. 5(a)] averages these values and makes it appear that two well-defined strain lobes emanate from the notch. These lobes are likely process zones of distinct matrix cracks and not a continuous region of high elastic and plastic strain in the silicon carbide. Further manipulation of the strain filter size (the subset size lower bound was constrained by the speckle size and distribution of the tracking pattern) applied to test HT-2 did not reveal additional matrix cracks. Also note that the displacement resolution of HT-1 was inherently less than that of HT-2 because, all other parameters being equal, it was obtained for a larger FOV.

It is beneficial to conduct analyses covering several tiers of noise filtering. In conducting both qualitative and quantitative analyses of damage in CMCs at small length scales, there appears to be no single combination of DIC parameters that yield optimal deformation field data. Over smoothing data can mask damage that would otherwise provide valuable insight into actual mechanical behavior. On the contrary, in the absence of sufficient filtering, high noise levels can mask real behavior.

Microscopically, the fracture behavior of HT-1 and HT-2 were quite different. Whereas a single crack originated from the notch in HT-2, two cracks emanated from the notch in HT-1, one of which branched into two additional matrix cracks. Small variations in microstructure and loading conditions may strongly influence when, where and how many cracks initiate at the notch as well as how they propagate. The notch root radius was as small as we could reasonably machine (precision methods like EDM were not feasible). A finer notch does not necessarily result in the initiation and propagation of a single crack with the fields of view we used. In Figs. 4 and 5 it is shown that the process zones ahead of the notch are small (hundreds of microns), so a notch length of $500 \mu \mathrm{m}$ is not likely to have a process zone that stretches to the other end of the gage width. Crack tip blunting was not a concern as the composites did not contain porous matrix phases.

\section{(2) High-Temperature Damage Evolution}

Accurate characterization of debond lengths in fibrous composites can be used to determine crack bridging tractions, which in turn can be incorporated into cohesive zone laws for damage modeling. In Fig. 6(a), debonding of the matrix crack along the length of longitudinal fibers is shown in two instances during test HT-2 (delineated with double arrows),

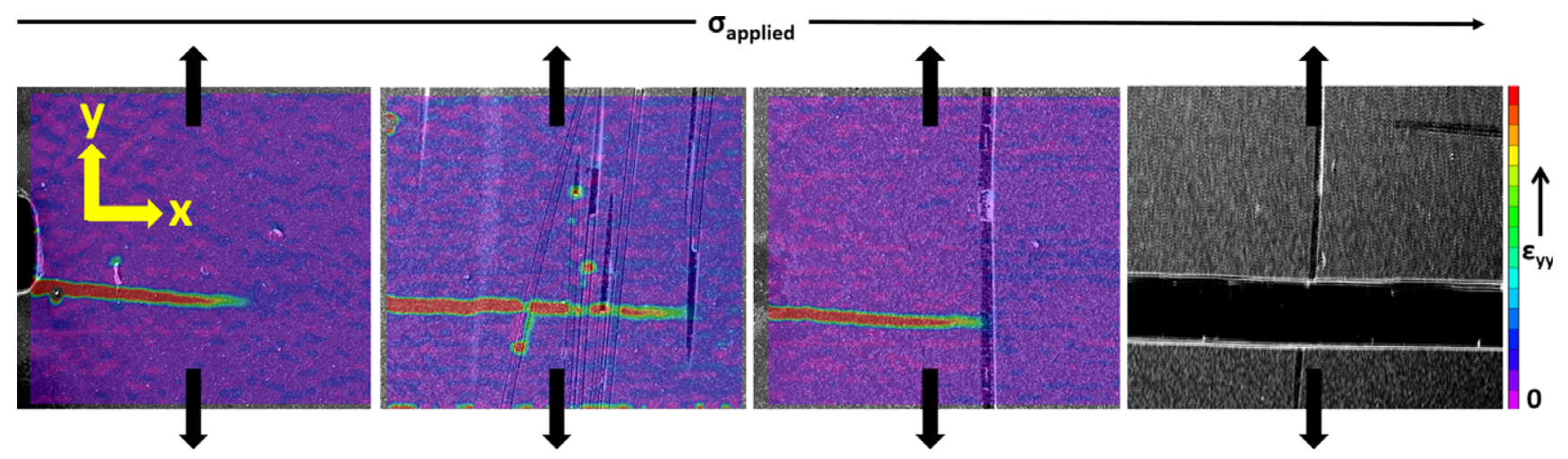

Fig. 3. Longitudinal strain fields along crack path. Each panel represents a shift in FOV as the crack propagates from left to right. The far right panel shows final fracture. Arrows indicate loading axis. 

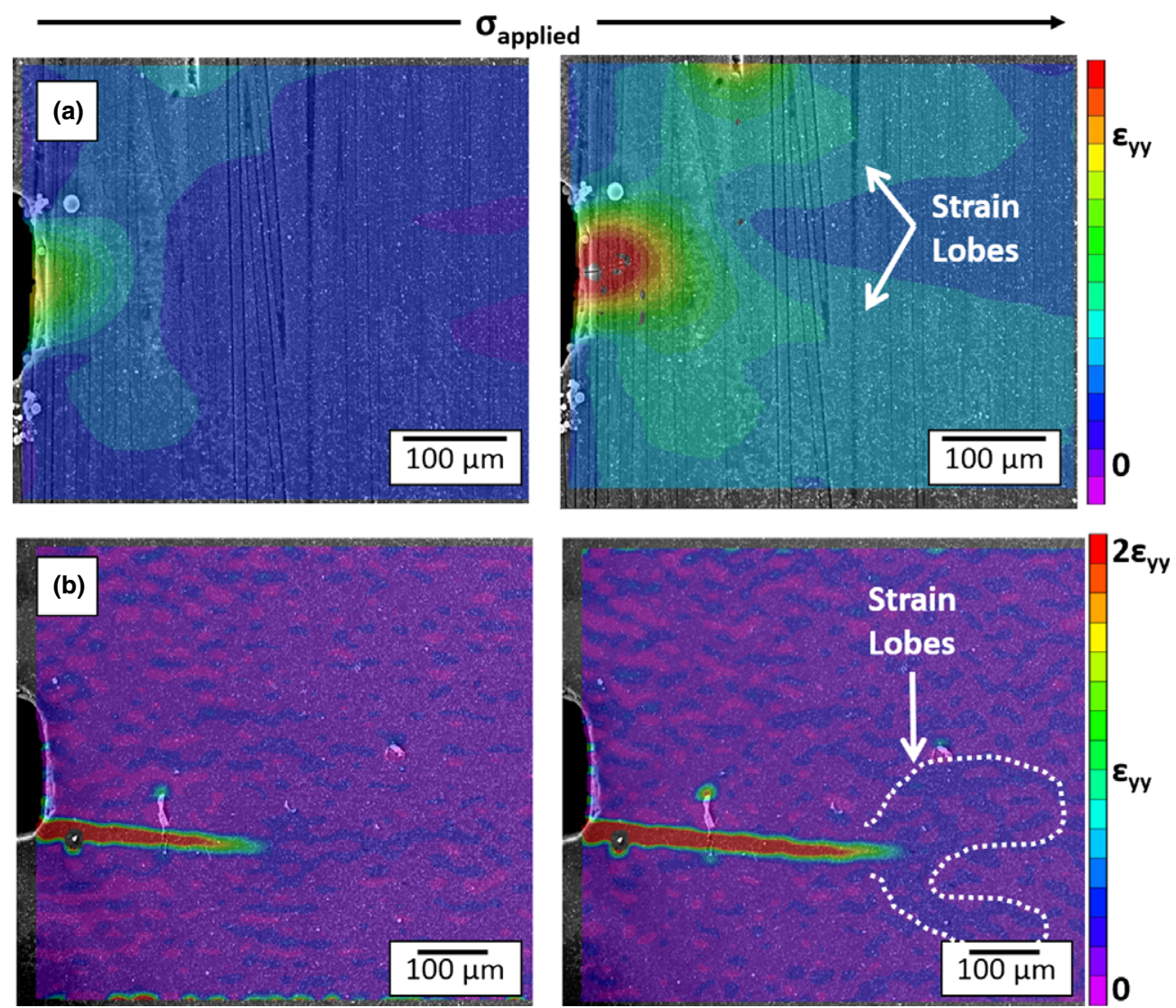

Fig. 4. Crack initiation (left) and propagation (right) in a $\mathrm{SiC} / \mathrm{SiC}$ composite at $795^{\circ} \mathrm{C}$ for FOVs of (a) 500 and (b) $800 \mu \mathrm{m}$. Longitudinal strain fields on left hand side correspond to the initial manifestation of surface matrix-crack at notch tip. Although both (a) and (b) exhibit high-strain process zones ahead of the crack tip, that of (a) appears larger. Note the different strain magnitude bars for (a) and (b).
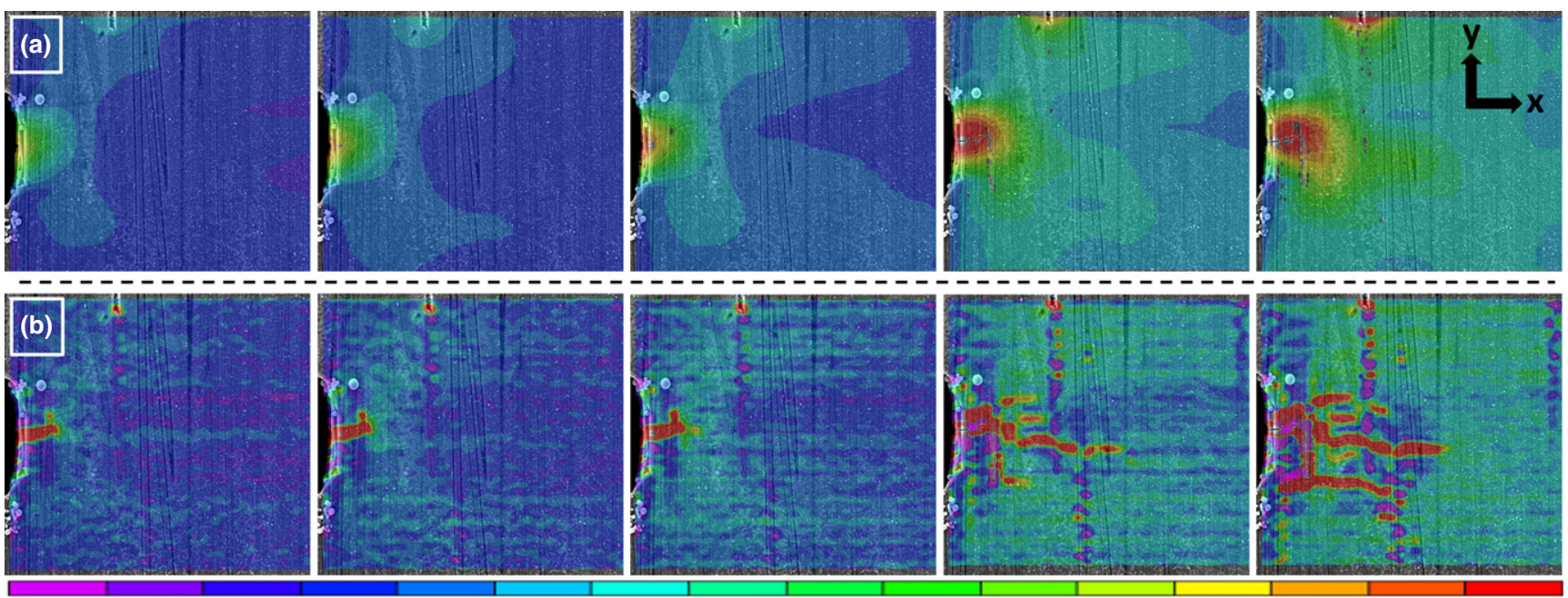

0

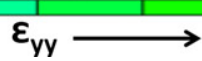

Fig. 5. Longitudinal strain fields illustrating the smearing effect of strain filters. Panel sequence indicates crack growth from left to right. In (a) the strain filter is $7 \times$ the subset size whereas in (b) it is $1.5 \times$ the subset size. The smaller strain filter does not mitigate artifacts of image noise and aliasing, however, it does reveal more damage, specifically multiple matrix cracks propagating from the notch tip, than the larger filter.

both with debond lengths of approximately $90 \mu \mathrm{m}$. Note that the exposed fibers were partially lapped and polished to prepare the specimen for testing; it is possible that the partial removal of fiber volume and damage to the exposed fibers may have influenced this debond length. Postfracture analysis of fiber pullout in the inner longitudinal ply nearest the coupon surface [Fig. 6(b)] revealed average pullout lengths of $72.7 \pm 29.4 \mu \mathrm{m}$. Although the $90 \mu \mathrm{m}$ debond lengths of [Fig. 6(a)] fall within this size distribution, a single ply-level distribution alone may not be reflective of the full distribu- tion of pullout lengths through the thickness of the laminate. Pullout data attained from DIC may be most useful when applied to the assessment of local toughening behavior at the CMC surface.

Fiber bridging tractions in the wake of a matrix crack strongly influence toughness. ${ }^{35}$ As ceramic fiber strengths are stochastic, both intact fibers that straddle the crack and ultimately fracture at the crack plane, as well as pullout fibers that break some distance away from the crack plane, contribute to toughening. To characterize the toughening contribution of 

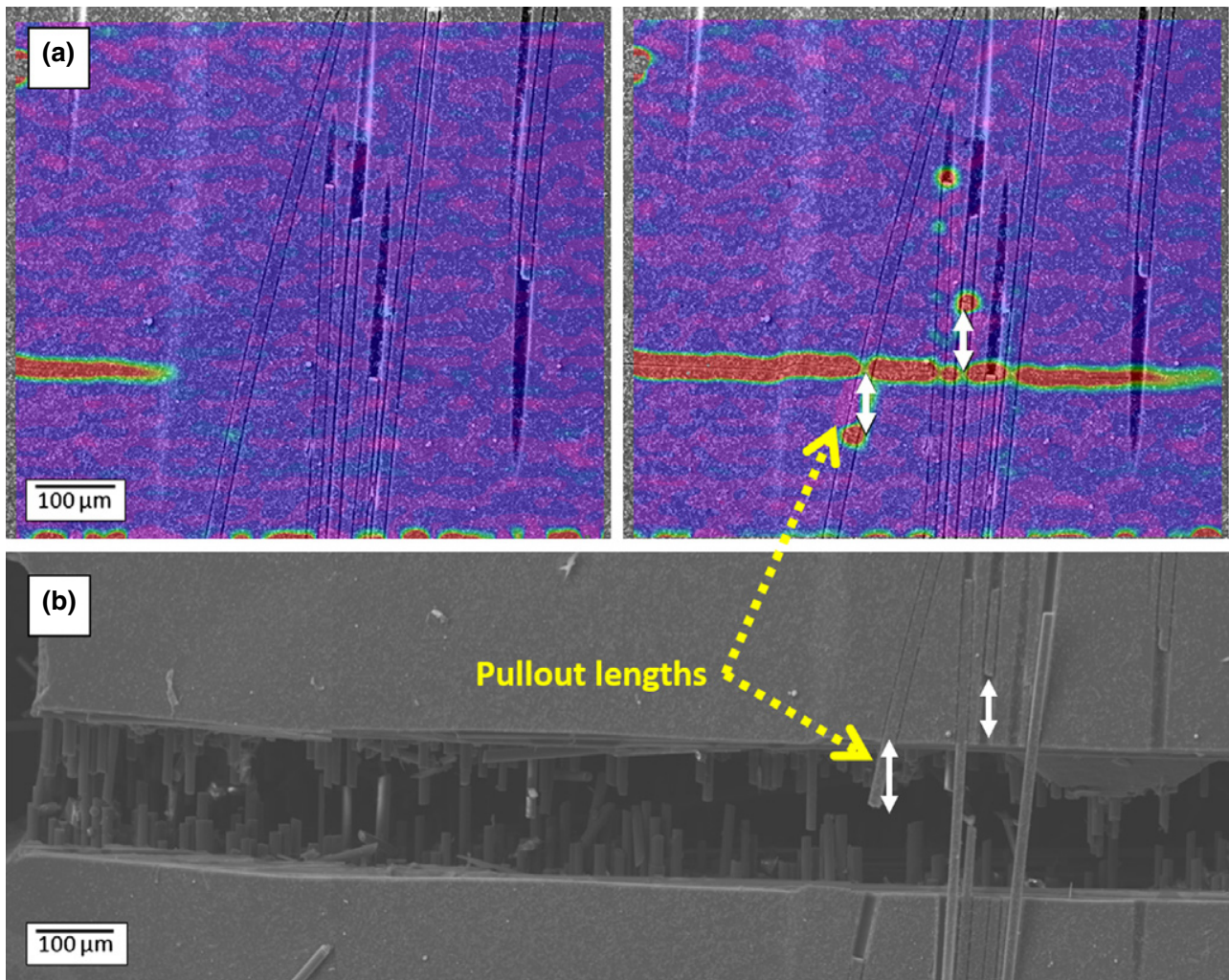

Fig. 6. Longitudinal strain fields depict debonding at the fiber-coating interface in the outer longitudinal lamina of the CMC (a). Fiber pullout lengths are of similar magnitude as debond lengths (b).

surface fibers that fracture away from the crack plane, full-field strain data from HT-2 was segmented into individual bins by microstructural feature, as illustrated in Fig. 7. Mean strains were computed for each fiber from the full-field strain data that fell within that fiber. Additional bins were created for the two fibers that exhibited pullout; mean strains in these bins included values that fell between the crack plane and break point of each fiber.

Mean fiber and pullout strains at each increment of crack propagation are plotted in Figure 8. Initial fiber breakage occurs in Fiber 1 (nearest the crack tip) at the third load increment, corresponding to a dip in the mean fiber strain. After the crack is introduced into the fiber, subsequent mean strains in both the fiber and fiber pullout bins increase as a result of the exaggerated stains computed near the crack. The exaggerated strains are an artifact of DIC measurements across a discontinuity. The minimum values may be due to relaxation in the fibers after fiber fracture. In reality, average strain should plateau or decrease. However, the exaggerated DIC measurements at the newly formed discontinuity result in large average strains that increase as the fiber cracks open. Up until this point, the mean strains in both the fiber and the pullout region are approximately half the theoretical failure strain of the fiber $\left(\varepsilon_{\mathrm{f}, \mathrm{u}}\right)$. Although the strain data is highly variable, the mean strain levels are within the expected strain range for this type of fiber.

The exaggerated strains that accompany fracture prevent using this strain data for a quantitative assessment fiber traction toughening. However, both fibers indicate a dip in mean strain at the point of fiber fracture. At that point, the error bars are at a minimum. Although further investigation is needed, dips in mean strains computed from DIC data may be indicative of ceramic fracture. Note that the negative ranges of standard deviation are indicative of noise in the DIC data.

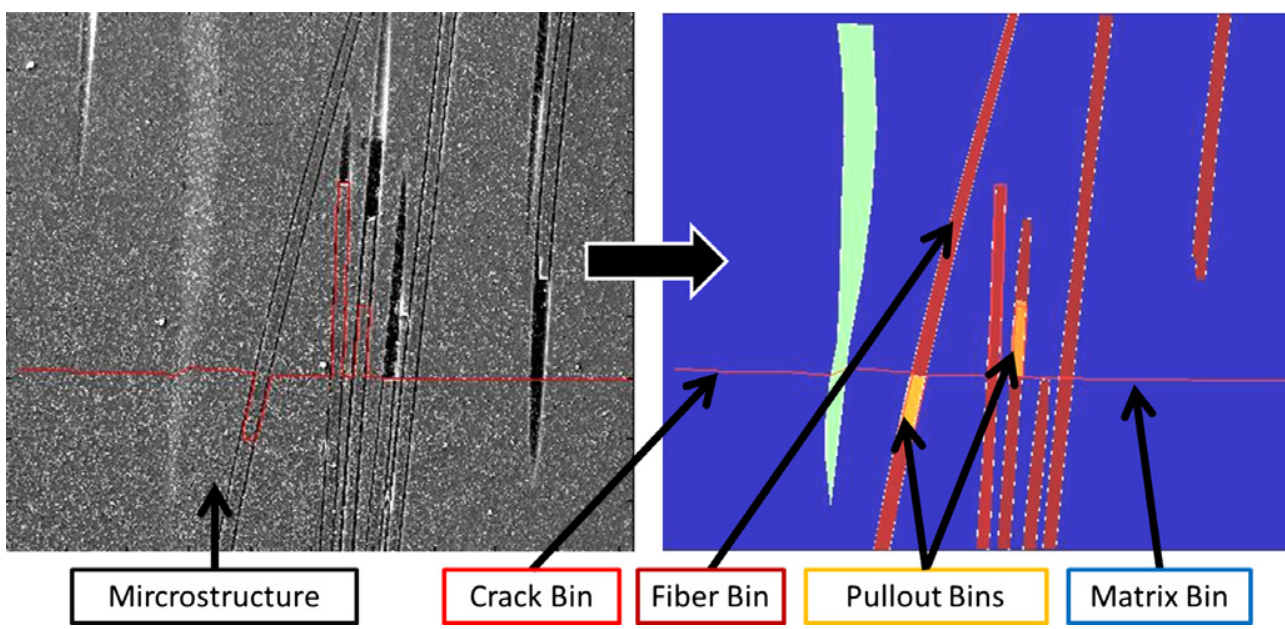

Fig. 7. Microstructure is segmented into fiber, fiber pullout, matrix, and crack bins. Mean strains are computed for each bin. 

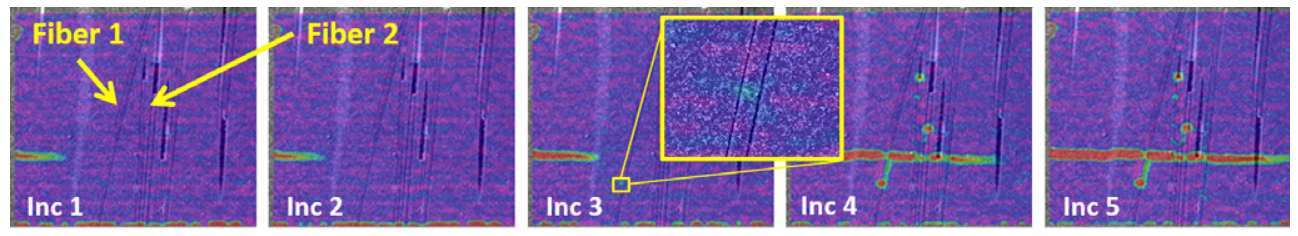

Fiber 1

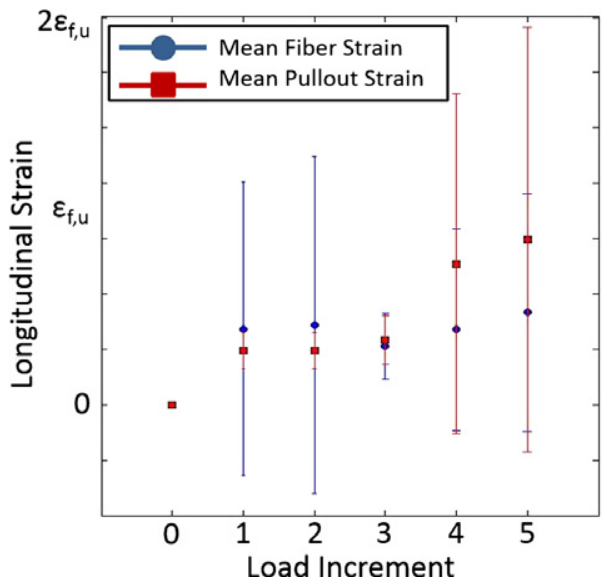

Fiber 2

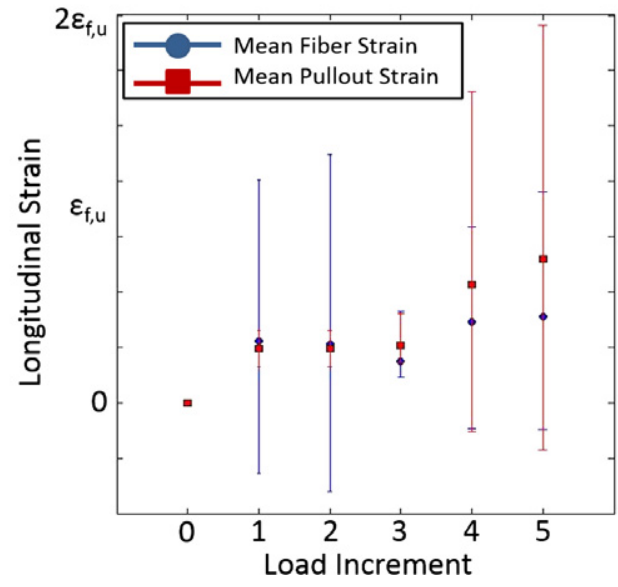

Fig. 8. Mean strains in fibers (computed from red and orange fiber bins) are plotted alongside their mean strains (computed from orange bins) in their corresponding pullout regions at each load increment. Inset in panel "Inc 3" is a magnified view of the localized tensile strain in a longitudinal fiber at fiber fracture.

Full-field strain analyses imply the following at the onset of fiber fracture:

1. Mean strains in the fiber increase during initial loading and then decrease to a minimum at fracture; and

2. Standard deviations in fiber/pullout strain were generally broad, but approached a minimum at fracture.

Although strain errors were large, mean strain measurements in the fibers fell within the range of what their failure strain would permit. Strain error must be carefully considered when conducting deformation field analysis to prevent drawing erroneous conclusions from trends in the dataset.

\section{(3) Sources of Error}

Aberrations in the full-field displacement data exist that introduce error into its quantitative analysis. These may be attributed to several sources of noise and bias. Noise in the DIC data is likely due to Gaussian noise inherent in the SEM images. Sources of bias may include debris and large aggregates on the speckle pattern, degradation in SEM image contrast with time, and aliasing. ${ }^{36}$ Gaussian noise was mitigated by utilizing $4 \times$ image integration for each image capture, which came at the expense of pixel dwell time (reduced from 100 to $10 \mu \mathrm{s}$ ) and thus image sharpness. Biasing effects were reduced using an optimized 8-tap interpolation filter. ${ }^{34}$ To further minimize error, deformation data in the vicinity of debris and large aggregates were not used for quantitative analysis. Aliasing, which can exist in portions of the pattern with speckles that encompass only a few pixels (as the corresponding subsets have lower fill factors), was mitigated using a low-pass filter. $^{36}$

Due to noise and biasing errors, it is necessary to conduct quantitative analyses of DIC-SEM data with great care and discretion. Differentiating displacements to get strain magnifies preexisting fluctuations, resulting in strain errors that can

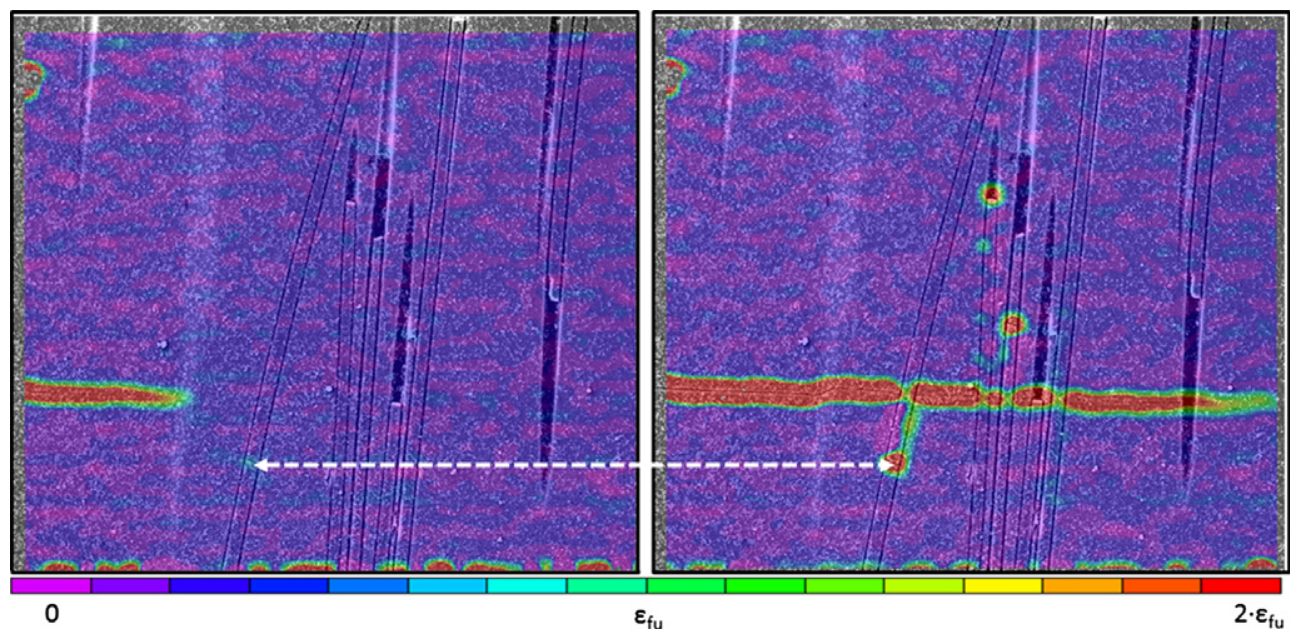

Fig. 9. Evolution of fracture in fiber. Double arrows point to position in fiber prior to and after fracture. Magnitude of longitudinal strain prior to fracture, as measured by DIC, is on order of the fiber's theoretical fracture strain. 
become larger than the material failure strain. In addition, when a crack forms it introduces an erroneously large displacement gradient that straddles both of its flanges. Consequently, the strain fields on either side of the crack may be several orders of magnitude larger than the actual physical values. These strain errors may mask real tensile strain in the material. However, it appears that DIC can resolve tensile strain in the ceramic fibers up to fracture, as shown in Fig. 9. Here, double arrows point to the location of fiber fracture just before and after fiber breakage. The DIC-measured strain values prior to fracture are near the known fracture strain value for the SiC fibers. The same cannot be said with regard to the matrix; the significantly lower fracture strain of the matrix results in noise that can mask the tensile strain. After the fiber fractures, the DIC-measured longitudinal strain increases by several orders of magnitude. Although this can be helpful in identifying cracks that can otherwise go undetected, care must be taken not to use these strain measures for quantitative analysis.

In future work these sources of error can be reduced through improved tracking patterns and use of higher quality SEMs. A tracking pattern composed of smaller or less agglomerated nanoparticles would permit use of a smaller FOV. Increasing the pixel density and using higher magnifications would then facilitate the use of smaller DIC subsets. Smaller subsets would increase spatial resolution, which would improve the signal to noise ratio of the full-field deformation measurements. Although some Gaussian noise may be inevitable, an SEM with a field emission gun (rather than a tungsten filament) would provide cleaner image data, which likewise would improve the signal to noise ratio.

\section{Conclusions}

A new experimental approach to investigate damage initiation and accumulation at the constituent length scale in CMCs under thermomechanical loading was evaluated. A colloidal system containing mechanically milled titanium nanopowder, bicine and water was developed for use as a DIC tracking pattern that is stable at temperatures in excess of $1000^{\circ} \mathrm{C}$. The patterning technique is adaptable to other high-temperature materials so long as the nanoparticles do not react with the substrate.

Strain fields captured in a notched CMC tensile coupon at $795^{\circ} \mathrm{C}$ in an SEM appeared to reveal a fluted process zone emanating from the notch tip. Reducing the data filter to increase spatial resolution, however, showed that this process zone consisted of several distinct matrix cracks emanating from the notch at different angles. Thus, full-field deformation maps must be interpreted with caution for CMCs as heavy data filtering and insufficient spatial resolutions can mask underlying damage.

Mean strains were computed in fibers at increasing load increments and appeared to show a drop in mean strain at fiber fracture. This is significant because implies that hightemperature SEM-DIC can measure linear elastic strain in the compliant fibers, which in turn can aid in quantifying toughening mechanisms in CMCs. However, there was significant strain error in the data, resulting in broad standard deviations. While DIC is a powerful to analyze deformation in ceramic materials, strain error must be carefully considered to prevent drawing erroneous conclusions from trends in the data.

\section{Acknowledgments}

We gratefully acknowledge Joshua Deboer for his assistance in developing the high-temperature testing technique discussed in this study and Dr. John Halloran for his helpful discussions. Experimental portions of this work were performed at the Electron Microbeam Analysis Laboratory (EMAL) at the University of Michigan.

\section{Supporting Information}

Additional Supporting Information may be found in the online version of this article:

Fig. S1. Ti nanopowder tracking patterns for high-temperature SEM-DIC experiments at a) 500 and b) $800 \mu \mathrm{m}$ fields of view. In both images, the tip of the notch is located on the left hand side. Variations in speckle density and the presence of a small number of abnormally large agglomerates are observed in both patterns.

Fig. S2. CMC mounted in load frame and fitted with heater $(\mathrm{S} 2 \mathrm{a})$. Double layer of tantalum heat shields with imaging aperture reduce thermal emissions $(\mathrm{S} 2 \mathrm{~b})$.

Fig. S3. (a) Chamber views of load frame, with heater and thermal shielding attached, in SEM at $23^{\circ} \mathrm{C}$ and $795^{\circ} \mathrm{C}$. At $795^{\circ} \mathrm{C}$ (right), thermal radiation from the heater is evident in its glowing case, but is contained by the shielding. (b) SEM and heat stage at $800^{\circ} \mathrm{C}$ with no thermal shielding.

\section{References}

${ }^{1}$ J. Aveston, G. A. Cooper, and A. Kelly, "Single and Multiple Fracture: The Properties of Fiber Composites"; (conference proceedings). IPC Science and Technology Press, Guilford, UK, 15-26 (1971).

${ }^{2}$ D. B. Marshall, B. N. Cox, and A. G. Evans, "The Mechanics of Matrix Cracking in Brittle-Matrix Fiber Composites," Acta Metall., 33, 2013-21 (1985).

${ }^{3}$ C. H. Hsueh, "Matrix Cracking with Frictional Bridging Fibres in Continuous Fibre Ceramic Composites," J. Mater. Sci., 30, 1781-9 (1995).

${ }^{4}$ B. Budiansky, J. W. Hutchinson, and A. G. Evans, "Matrix Fracture in Fiber-Reinforced Ceramics," J. Mech. Phys. Sol., 34 [2] 167-89 (1986).

${ }^{5}$ P. F. Becher, C. H. Hsueh, P. Angelini, and T. N. Tiegs, "Toughening Behavior in Whisker-Reinforced Ceramic Matrix Composites," J. Am. Ceram. Soc., 71 [12] 1050-61 (1988).

${ }^{6}$ F. W. Zok and C. L. Hom, "Large Scale Bridging in Brittle Matrix Composites," Acta Metall. Mater., 38 [10] 1895-904 (1990).

${ }^{7}$ K. G. Dassios and C. Galiotis, "Direct Measurement of Fiber Bridging in Notched Glass-Ceramic Matrix Composites," J. Mater. Res., 21 [5] 1150-60 (2006).

${ }^{8}$ M. A. Sutton, J. Orteu, and H. W. Schreier, Image Correlation for Shape, Motion and Deformation Measurements: Basic Concepts, Theory and Applications. Springer, New York, NY, 2009.

${ }^{9}$ M. A. Sutton, M. Cheng, W. H. Peters, Y. J. Chao, J. Orteu, and S. R. McNeill, "Application of an Optimized Digital Correlation Method to Planar Deformation Analysis," Image Vision Comput., 4 [3] 143-53 (1986).

${ }^{10}$ M. A. Sutton, N. Li, D. Garcia, N. Cornille, J. Orteu, S. R. McNeill, H. W. Schreier, and X. Li, "Metrology in a Scanning Electron Microscope: Theoretical Developments and Experimental Validation," Meas. Sci. Technol., 17, 2613-22 (2006).

${ }^{11}$ M. A. Sutton, N. Li, D. C. Joy, A. P. Reynolds, and X. Li, "Scanning Electron Microscopy for Quantitative Large Deformation Measurements Part I: SEM Imaging at Magnifications from 200 to 10,000," Exp. Mech., 47, 77587 (2007).

${ }^{12}$ M. A. Sutton, N. Li, D. Garcia, N. Cornille, J. J. Orteu, S. R. McNeill, H. W. Schreier, A. P. Reynolds, and X. Li, "Scanning Electron Microscopy for Quantitative Large Deformation Measurements Part II: Experimental Validation for Magnifications from 200 to 10,000," Exp. Mech., 47, 789-804 (2007).

${ }^{13}$ A. D. Kammers and S. Daly, "Digital Image Correlation Under Scanning Electron Microscopy: Experimental Methodology and Validation," Exp. Mech., 53 [174] 3-1761 (2013).

${ }^{14}$ L. P. Canal, C. Gonzalez, J. M. Molina-Aldareguia, J. Segurado, and J. Llorca, "Application of Digital Image Correlation at the Microscale in Fiber-Reinforced Composites," Compos. Part A (Appl. S.), 43 [10] 1630-8 (2012).

${ }^{15}$ A. D. Kammers and S. Daly, "Small-Scale Patterning Methods for Digital Image Correlation Under Scanning Electron Microscopy," Meas. Sci. Technol., 22, 125501, 12pp (2011).

${ }^{16}$ W. A. Scrivens, Y. Luo, M. A. Sutton, S. A. Collete, M. L. Myrick, P. Miney, P. E. Colavira, A. P. Reynolds, and X. Li, "Development of Patterns for Digital Image Correlation Measurements at Reduced Length Scales," Exp. Mech., 47, 63-77 (2007).

${ }^{17}$ S. A. Collete, M. A. Sutton, P. Miney, A. P. Reynolds, X. Li, P. E. Colavita, W. A. Scrivens, Y. Luo, T. Sudarshan, P. Muzykov, and M. L. Myrick, "Development of Patterns for Nanoscale Strain Measurements: I. Fabrication of Imprinted Au Webs for Polymeric Materials," Nanotechnology, 15, 1812-7 (2004)

${ }^{18}$ N. Thaulow and E. W. White, "General Method for Dispersing and Disaggregating Particulate Samples for Quantitative SEM and Optical Microscopic Studies," Powder Technol., 5, 377-9 (1971)

${ }^{19}$ A. D. Kammers and S. Daly, "Self-Assembled Nanoparticle Surface Patterning for Improved Digital Image Correlation in a Scanning Electron Microscope," Exp. Mech., 53, 1333-41 (2013). 
${ }^{20}$ V. P. Rajan, M. N. Rossol, and F. W. Zok, "Optimization of Digital Image Correlation for High-Resolution Strain Mapping of Ceramic Composites," Exp. Mech., 52, 1407-21 (2012).

${ }^{21}$ M. D. Novak and F. W. Zok, "High-Temperature Materials Testing with Full-Field Strain Measurement: Experimental Design and Practice," Rev. Sci. Instr., 82, 115101, 6pp (2011).

${ }^{22}$ R. G. Freeman, K. C. Grabar, K. J. Allison, R. M. Bright, J. A. Davis, A. P. Guthrie, M. B. Hommer, M. A. Jackson, P. C. Smith, D. G. Walter and M. J. Natan "Self-Assembled Metal Colloid Monolayers: An Approach to SERS Substrates," Science, 267, 1629-32 (1995).

${ }^{23}$ K. C. Grabar, R. G. Freeman, M. B. Hommer, and M. J. Natan, "Preparation and Characterization of Au Colloid Monolayers," Anal. Chem., 67, 735-43 (1995)

${ }^{24}$ K. C. Grabar, K. J. Allison, B. E. Baker, R. M. Bright, K. R. Brown, R. Griffith Freeman, A. P. Fox, C. D. Keating, M. D. Musick and M. J. Natan, "Two-Dimensional Arrays of Colloidal Gold Particles: A Flexible Approach to Macroscopic Metal Surfaces," Langmuir, 12 [10] 2353-61 (1996).

${ }^{25}$ M. J. Hudson, J. W. Peckett, and P. J. Harris, "Low-Temperature Sol-Gel Preparation of Ordered Nanoparticles of Tungsten Carbide/Oxide," Ind. Eng. Chem. Res., 44 [15] 5575-8 (2005).

${ }^{26} \mathrm{O}$. M. Nayfeh, D. A. Antoniadis, K. Mantey, and M. H. Nayfeh, "Uniform Delivery of Silicon Nanoparticles on Device Quality Substrates Using Spin Coating from Isopropyl Alcohol Colloids," Appl. Phys. Lett., 94, 1-3 (2009).

${ }^{27}$ C. Giordano, W. Yang, A. Lindemann, R. Crombez, and J. Texter, "Waterborne WC Nanodispersions," Colloid Surface A, 374, 84-7 (2011).

${ }^{28}$ E. Laarz and L. Bergstrom, "Dispersing WC-Co Powders in Aqueous Media with Polyethylenimine," Int. J. Refract. Met. H, 18 [6] 281-6 (2011).
${ }^{29}$ A. M. Elbadawy, T. P. Luxton, R. G. Silva, K. G. Schekl, M. T. Suidan, and T. M. Tolymat, "Impact of Environmental Conditions ( $\mathrm{pH}$, Ionic Strength, and Electrolyte Type) on the Surface Charge and Aggregation of Silver Nanoparticles Suspensions," Environ. Sci. Technol., 44, 1260-6 (2010).

${ }^{30}$ A. Godara and D. Raabe, "Microstrain Localization Measurement in Epoxy FRCs During Plastic Deformation Using a Digital Image Correlation Technique Coupled with Scanning Electron Microscopy," Nondestrcut. Test Eva, 23 [3] 229-40 (2008).

${ }^{31}$ S. P. Lee, H. K. Yoon, J. S. Park, Y. Katoh, and A. Kohyama, "Processing and Properties of $\mathrm{SiC}$ and $\mathrm{SiC} / \mathrm{SiC}$ Composite Materials by Melt Infiltration Process," Int. J. Mod. Phys. B, 17 [8-9] 1833-8 (2003).

${ }^{32}$ K. L. Luthra and G. S. Corman, "Melt Infiltrated (MI) SiC/SiC Composites for Gas Turbine Applications"; pp. 744-53 in High Temperature Ceramic Matrix Composites, Edited by W. Krenkel, R. Naslain, and H. Schneider. Wiley-VCH, Weinheim, Germany, 2001.

${ }^{33}$ K. L. Luthra, R. N. Singh, and M. K. Brun, "Toughened Silcomp Composites - Process and Preliminary Properties," Am. Ceram. Soc. Bull., 72 [7] 79-85 (1993).

${ }^{34}$ Correlated Solutions, VIC-2d [Software]. Correlated Solutions, Columbia, SC, 2009.

${ }^{35}$ M. D. Thouless and A. G. Evans, "Effects of Pull-out on the Mechanical Properties of Ceramic-Matrix Composites," Acta Metall., v, 36 [3] 517-22 (1988).

${ }^{36}$ Correlated Solutions, Advanced Bias. http://correlatedsolutions.com/support/, 2011. 